\title{
Research on the Design of the General Hospital Wayfinding and Signage System based on the Universal Design Concept - A Case Study of the Second Affiliated Hospital of Nanchang University
}

\author{
Xinbo Shen ${ }^{1, *}, \mathrm{Min} \mathrm{Li}^{1}$ and Lei $\mathrm{Yu}^{1}$ \\ ${ }^{1}$ Art College, Jiangxi University of Finance and Economics, Nanchang City, Jiangxi Province, 330013, China
}

\begin{abstract}
With the continuous improvement of the medical level in China, the quality of hospital service and the environment have attracted people's attention. The hospital is a place with relatively dense population, and the general hospital consists of a number of departments with complicated procedures and staggered routes. In order to improve the efficiency of medical treatment, stabilize the mood of patients, and respond to the call of establishing a human-oriented hospital, it is particularly important to design a reasonable and comprehensive hospital wayfinding and signage system. In this paper, the universal design concept and principles which are more suitable for everyone are applied to the hospital wayfinding and signage system. Through the field investigation of the Second Affiliated Hospital of Nanchang University, the author analyzes the problems and sums up the design criteria which are suitable for the hospital wayfinding and signage system.
\end{abstract}

\section{Introduction}

Universal design is produced on the basis of barrier-free design and is an extension of the human-oriented concept. It refers to the design of the environment, products and services that can be used by anyone without special design or adjustment[1]. It is not designed specifically for a certain kind of people, and it is not influenced by the economy, education, status, ability and other factors. It is a comprehensive design to truly realize the equality of all people. Children, adults, the elderly and people with disabilities can all benefit from it. Universal design has the characteristics of inclusiveness, convenience, comfort and flexibility. It is applicable to all fields and all people. It covers most groups and integrates individual differences. It is additive design, which can meet people's more accurate and simple use requirements, and make visual, tactile, auditory and other effects better.

Hospital is a place closely related to people. In recent years, the medical environment and service level in our country have gradually increased. For a general hospital, the flow of people is large, the action line is complex, and there are many medical procedures. In order to get treatment safely, quickly and effectively, the wayfinding and signage system must be clear, scientific and humanized. The earliest research on hospital wayfinding and signage system in China dates back to 2004 when the research was mainly about the aspects of humanistic care, interest and functionality. For example, Fu Tiantian, from the Qilu University of Technology, put forward the method, principle and implementation of the design of hospital wayfinding and signage system based on human in Research on "Human-oriented" Design in Visual Wayfinding and Signage System of Hospital. Chen Le, from Anhui University of Engineering finished the Research on the Individualized Design of Modern Medical Environment Wayfinding and Signage System. In this paper, he explores the methods, processes and principles of hospital individualized design, and makes case analysis of municipal hospitals, which involves less universal design theory. Through field and literature research, together with analysis and induction, the author mainly analyzes the deficiencies of the wayfinding and signage system of the general hospital - the Second Affiliated Hospital of Nanchang University. The universal design is used to optimize its wayfinding and signage system, to improve the difficulty of people's medical treatment caused by the lack and confusion of wayfinding and signage, to enable patients to arrive at the destination clearly, quickly and accurately, to improve the service quality and image of the hospital, and to provide reference for the design and application of the wayfinding and signage system of the general hospital.

\section{$2 \quad$ The wayfinding and signage system of general hospital and its classification}

*Corresponding author e-mail: 870664319@qq.com 


\subsection{Concept of general hospital wayfinding and signage system}

The general hospital has a wide range of targeted patients which sets up a number of specialized projects composed of internal and external, traditional Chinese and western medical departments with its sections focusing on maternal and child, ear, nose and throat[2]. The wayfinding and signage system of the hospital is a wayfinding and signage system that uses the physical properties of materials and colors, as well as various text and graphic signs to guide the logistics and people flow within or around the hospital[3]. A good, standardized and human-oriented hospital wayfinding and signage system can guide patients and their families to find their way quickly and accurately. It can also guide patients to see a doctor in time with the improving working efficiency of medical staff, and stabilizing the working order of the hospital. It is an essential hardware facility of the hospital as well as a miniature of the image of the hospital.

\subsection{Classification of wayfinding and signage system of general hospital}

Accurate information classification is the key to the establishment of hospital wayfinding and signage system. In the document Code for Architectural Design of General Hospitals issued by China, it clearly points out that the hospital wayfinding and signage system is divided into four levels: (1) the first-level wayfinding and signage system: it is mainly the wayfinding and signage outside hospitals or on the building including building name, entrance and exit, together with general layout, road diversion, public service, and the overall image and other signs; (2) the second-level wayfinding and signage system: it mainly includes channel and floor sign, including index and plan of each floor, guidance of step elevator, expert column, visit form, entrance and exit, etc; (3) the third-level wayfinding and signage system: it mainly involves the sign of functional areas, including the sign of medical care, administrative logistics and other units; (4) the forth-level wayfinding and signage system: it mainly refers to room window and doorplate, including each ward, sickbed, department and other doorplate window plates. The classification of hospital wayfinding and signage system can make the system more standardized, avoid the waste of resources, and make the division of labor in the design process clearer.

\section{Problems in the wayfinding and signage system of the Second Affiliated Hospital of Nanchang University}

The Second Affiliated Hospital of Nanchang University is a comprehensive 3A hospital in Jiangxi Province, which integrates medical treatment, scientific research and rehabilitation. It has more than 2000 beds and 51 departments. It is mainly divided into three main buildings: outpatient department complex building, inpatient department and physical examination building, as well as canteen and office building. Its large scale and complex distribution highlight the importance of a proper guide wayfinding and signage system. Based on the field investigation of the Second Affiliated Hospital, the author finds out the problems in the practical application of the wayfinding and signage system, which are summarized as follows:

\subsection{The imperfect wayfinding and signage system and the lack of planning}

A complete, standardized and integrated wayfinding and signage system plays an important role in the complex hospital environment. If the system fails to perform the function, patients and their families are unable to get information signs and will be left in confusion. There is no standard wayfinding and signage system inside and outside the Second Affiliated Hospital, which is mainly reflected in the disordered and crowded wayfinding sign and its irregular placement. Some specific hints are not given. The signs of outpatient department, comprehensive building and inpatient department are blue, while the physical examination area of female guests in the physical examination building is red, and the radiotherapy area is green, 'figure 1'; there is only a simple planar graph in the entrance of the hospital instead of index plan of the whole hospital. There is no any floor guidance in the physical examination building and the comprehensive building; some signs are placed on the ground or near stairway in disorder without standard size or forms, 'figure 2'. Also, the warning sign is inadequate and fuzzy, which is not easy to find in walking.

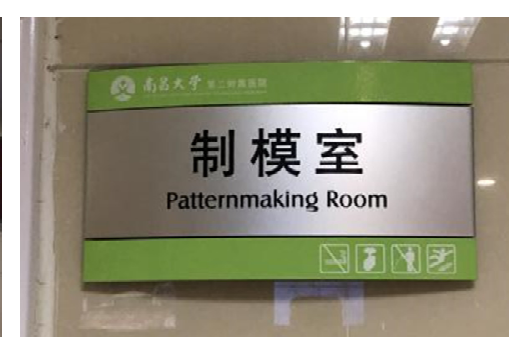

Figure 1. The wayfinding and signage system of female physical examination area and radiotherapy area in the physical examination building of the Second Affiliated Hospital of Nanchang University. 

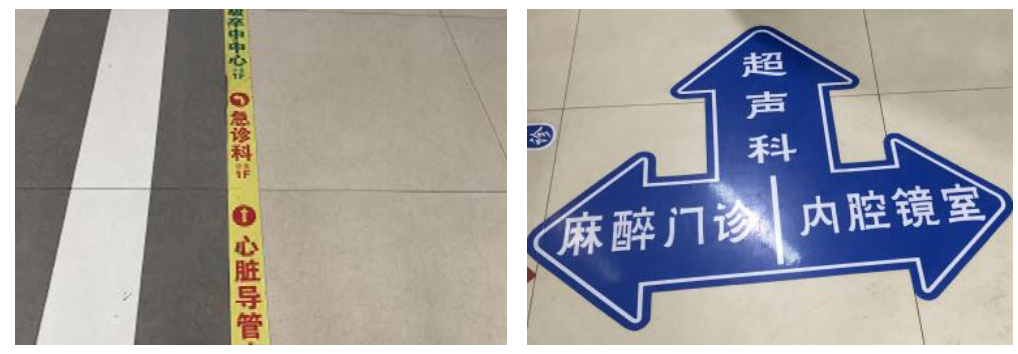

Figure 2. The ground wayfinding and signage system of the inpatient department and multi-functional building of the Second Affiliated Hospital of Nanchang University.

\subsection{Unreasonable sign setting and fuzzy direction}

Unnecessary troubles can be prevented with appropriately located signs and clear directions when people seek medical treatment. The problems of the Second Affiliated Hospital appear are mainly reflected in the fact that patients are difficult in finding the designated area because of unclear signs. For example, when one go to pediatrics obeying the sign, he will get to surgery; besides, the location of some signs are too high, such as department wayfinding and signage, floor guidance at stairs and the guidance of inpatient department, whereas some outdoor wayfinding and signage are so lower to the ground that vehicle can easily block them, making these signs inconvenient to identify; in addition, there are no signs in the places where the flow of people is large and chaos is easy to cause, and the

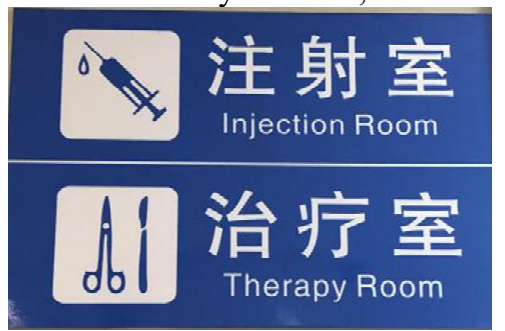

process of medical treatment is not considered in the setting of signs.

\subsection{Lack of aesthetics and design sense}

The hospital's wayfinding and signage system should not only be intuitive and clear, but also pay attention to the combination of the three elements of graphics, color and text so as to show the aesthetic sense and significance of the wayfinding and signage. The problems of the Second Affiliated Hospital are mainly reflected on the facts that some fonts are too small to recognition, especially when the fonts are all in bold type without distinction; the wayfinding and signage on the graphics are often arrows, and the logo patterns are international style without unique graphic signs or creativity, 'figure 3'; although most of the colors are blue, they are in different shades and some parts are even red and green, which are in disorder and lack aesthetics.

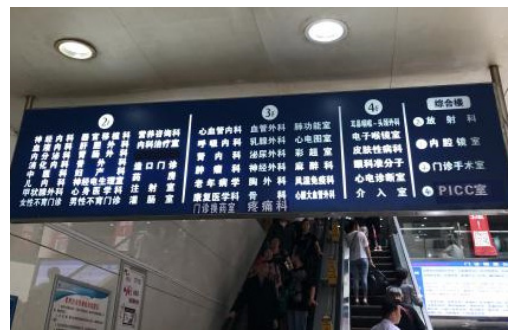

Figure 3. The wayfinding and signage system of the outpatient departments of the Second Affiliated Hospital of Nanchang University.

\subsection{Lack of human-oriented awareness}

The aging of our country is gradually increasing with more and more people suffering from disability and color blindness. For the special group of people, the hospital wayfinding and signage system should be human-oriented and comprehensive. The Second Affiliated Hospital is a large-scale comprehensive hospital located in the center of the city, consisting of a large number of people who live in counties, districts and towns. Each of them has a different education level. However, the hospital does not provide illiterate signs for people with limited literacy; as a special group, most of the children are afraid when seeking medical treatment, but the design of the signs for pediatrics lacks interest and flexibility, which is not helpful for children to overcome anxiety in diagnosis and treatment. The tactile signs for disabled patients and some signs for wheelchair users are not offered in the hospital, such as the specific height and proportion of corridors and elevators, making people difficult to get accurate information of the wayfinding and signage.

\section{$4 \quad$ Universal design of the wayfinding and signage system of the Second Affiliated Hospital of Nanchang University}

Although the wayfinding and signage system of the Second Affiliated Hospital has played a guiding role in reality, there are still many problems to be improved. The design of wayfinding and signage system in hospital is complicated. It is not easy to adapt to different groups of people and to be used by all people. Therefore, it is necessary to apply the universal design theory to solve the problem of wayfinding and signage system, and fully consider the needs and psychology of all kinds of people. Based on the literature review and the analysis of the problems of the wayfinding and signage of the Second 
Affiliated Hospital, three principles for the design of the wayfinding and signage system of the hospital are summarized:

\subsection{Integration and rationalization}

The wayfinding and signage system of the hospital shall be complete and systematic, conform to the relevant national standards, and the wayfinding and signage of all regions and types shall be unified, and be consistent with the hospital's own culture and style, such as the font size, font, spacing and typesetting of the characters, and the color system, material, form, layout, size, location and graphic design of the signs at all levels shall be unified, concise and beautiful. Besides, the identification of special prompt shall be obvious. The setting of wayfinding and signage should be clearly divided into four levels, so that patients can be guided to each functional area according to the indoor and outdoor wayfinding and signage. On this basis, the scale of signs at all levels should also be reasonably set, taking into account the spatial relationship, floor height, visual comfort of people in different postures and body shapes. For example, Budapest Airport has set up a series of special reasonable signs beside the normal size signs to remind wheelchair users and children.

\subsection{Easy to distinguish with accuracy}

The perfect layout of hospital wayfinding and signage shall be accurate. According to the flow of people, medical treatment process and building characteristics, the appropriate placement position, size and frequency shall be calculated to avoid the absence of signs and the occlusion caused by height. The ground, wall, ceiling and other forms shall be selected at the reasonable position to enhance the clarity. The medical wayfinding and signage system should be in consistency and relevance. It is necessary to set up corresponding bifurcated wayfinding and signage at the turning of the intersection and special areas[4], such as elevator stairs, entrances and exits, so as to prevent the delay of diagnosis and treatment due to route deviation, ensure the accuracy of orientation and information, and indicate the destination. Secondly, the information of logo should be well arranged according to importance. Also, when typesetting, important information should be highlighted, omitting unnecessary details. Chinese and English words should be used accurately. Graphics should be matched with words. The size, proportion, color and brightness of light should be compared. The visual background should be simple and intuitive, easy to understand and reduce confusion caused by ambiguous content.

\subsection{Humanized and green design}

The human-oriented medical service is improved constantly in which patients are regarded as the center of the hospital. The hospital wayfinding and signage system designed for people can optimize the service level and improve the diagnosis and treatment environment.
Considering the complexity of the hospital population, corresponding signs should be set up at home and abroad to meet different needs for old children, defective patients and family members, medical staff, etc. The book Design in Design written by Kenya Hara refers to the theory of "five senses" that integrates vision, touch, hearing, smell and taste into the design[5], which can transmit information in various ways, allowing people to look up information from different aspects, and add voice and Braille prompts to the literal wayfinding and signage. When using the wayfinding and signage, the adjustment of people's actions should be reduced as much as possible, with more attention to the safety, and the appearance of sharp and fragile materials shall be avoided. Also, information in the dangerous area should be put noticeably and the risk coefficient should be reduced. Green design has gradually become the mainstream. Hospital wayfinding and signage can be made of environmental friendly materials instead of traditional materials, which are durable, recyclable, cost-effective, and easy to change. Moreover, they can transmit emotions through the selection and use of materials. For example, Meitian hospital, a Japanese specialized hospital for women and children breaks through the traditional concept of industrial materials and uses soft white pure cotton cloth to make signs in shape of everyday products. In this way, affinity is increased, fear eliminated, a clean, warm and comfortable image of the hospital displayed[6]. Such signs are designed from the perspective of human care.

\section{Conclusion}

The service quality of the hospital has been a prior concern in the life of the public. A well-established hospital wayfinding and signage system helps to save more time in saving patients' lives and provide convenience for medical treatment. The Second Affiliated Hospital of Nanchang University is a 3A comprehensive hospital with many departments, complex processes and transportation, and it also undertakes various duties like high-level medical treatment, scientific research, practical teaching and other tasks. This paper summarizes the existing problems of the Second Affiliated Hospital's wayfinding and signage system, such as lack of planning and unreasonable setting. The paper applies the universal design theory to real problems and puts forward three principles for improvements which can promote practical problems such as humanization and systematization with the explanation of relevant concepts. The author hopes the paper serves as a reference for the design of similar hospitals' wayfinding and signage systems.

\section{References}

1. Zeng, L.X., Jiang, X. (2014) Concept analysis of universal design. CAD / CAM and Manufacturing Informatization, 7: 25-26. 
2. Chu, L.F. (2005) Research on the design of wayfinding sign recognition system of general hospital. Jiangnan University, 8-9.

3. Wang, W.Z. (2013) Research and design of Z Hospital wayfinding and signage system . Hebei University of Technology, 12-13.

4. Wang, L. (2007) Research on humanized design of medical wayfinding and signage system. Hefei University of Technology, 24-25.

5. Kenya, H. (2006) Design in Design. Shandong People's Publishing House, Jinan.

6. Li, G., Li, W.Y. (2015) Analysis of emotional design in Meitian Hospital's wayfinding and signage system. Design, 1: 92-93.

7. Li, M. (2015) Research on the design method of urban public environment wayfinding and signage system - Taking Matsushan City, Japan as an example. New arts, 5: 120-124.

8. Li, M. (2014) Research on the design of urban public environment wayfinding and signage system. New arts, 7: 109-110.

9. Li, M., Hu, Y. (2012) Research on the design method of urban wayfinding and signage system. New arts, 5: 68-72.

10. Wu, X.X. (2015) Design and research on passenger flow wayfinding and signage system of medical institutions. Donghua University, 45-47. 